\title{
Gradual Neurologic Deterioration Post Kyphoscoliosis Correction Surgery: A Case Report
}

\author{
Jea Woo Lim, Veushj Sharma, Hak Sun Kim
}

Department of Orthopaedic Surgery, Gangnam Severance Hospital, Yonsei University College of Medicine, Seoul, Korea

A 13-year-9-month-old female child presented with congenital kyphoscoliosis along with progressive paraparesis. Radiographs confirmed kyphoscoliosis and magnetic resonance imaging revealed a stretched and flattened spinal cord over the kyphotic deformity and a T7 hemivertebra. She underwent a posterior correction of the curve along with posterior decompression and a posterior to anterior excision of T7 hemivertebra to relieve her of the deteriorating neurology. While carrying out the excision of T7 hemivertebra, her trans cranial electrical motor evoke potential dropped. Consequently, she was administered a mega dose steroid therapy. After a positive wake-up test, the excision was discontinued and surgery was concluded by in situ fixation of the deformity with short rods. Thereafter, a gradual deterioration in the neurologic status was observed and patient became paraplegic on the fourth post operative day. In this case report, we try to analyze various causes for gradual deterioration in neurologic status.

Key Words: Kyphosis, Paraparesis, Paraplegia, Motor evoked potentials

\section{Introduction}

Congenital kyphoscoliosis accounts for $10 \%$ of all scoliosis, which require treatment [1]. Congenital kyphosis and kyphoscoliosis are potentially the ones, which experience a rapid curve progression and hence need to be corrected at an earlier age. In fact it has been postulated in a retrospective study that congenital kyphosis/kyphoscoliosis needs to be addressed surgically before the age of 5 years and before the kyphosis exceeds $50^{\circ}$ [2]. If the condition is left uncorrected, the progression can deteriorate the neurological status of the individual due to mechanical strain over the spinal cord or due to some associated local pathology. Besides, cosmetic disfigurement is another aspect, which at times becomes a necessary indication for the corrective surgery. While attempting the correction manoeuvre in spinal deformities, many a times some unforeseen inexplicable incidences can occur that may lead to a postoperative deteriorated neurological status. Our present endeavour is to present a case report wherein a child having congenital kyphoscoliosis with paraparesis underwent posterior correction of curve with instrumentation, posterior decompression, excision of hemivertebra and anterior interbody fusion to relieve her of neurologic symptoms; later she gradually turned paraplegic. We also try to explore the possible causes of such unwanted end result.

\section{Case Report}

A 13-year-old female child complained of back pain, weakness in the left lower extremity and difficulty in walking. At that time, she had scoliosis from T5 to T10 and her kyphotic angle was $65.4^{\circ}$. She was advised to wear Milwaukee brace. During a follow up visit (9 months later), her complaints of back pain had become more pronounced and difficulty in walking had aggravated. Radiologic examination revealed curve progression despite the presence of brace. At this time, the kyphotic angle had increased to

Received Dec 18, 2011; Revised Mar 7, 2012; Accepted Mar 14, 2012

Corresponding author: Hak Sun Kim, MD

Department of Orthopaedic Surgery, Gangnam Severance Hospital,

211 Eonju-ro, Gangnam-gu, Seoul 135-720, Korea

Tel: +82-2-2019-3411, Fax: +82-2-573-5393, E-mail: haksunkim@yuhs.ac

Copyright (C) 2012 by Korean Society of Spine Surgery

This is an Open Access article distributed under the terms of the Creative Commons Attribution Non-Commercial License (http://creativecommons.org/licenses/by-nc/3.0/) which permits unrestricted non-commercial use, distribution, and reproduction in any medium, provided the original work is properly cited. Asian Spine Journal • pISSN 1976-1902 eISSN 1976-7846 

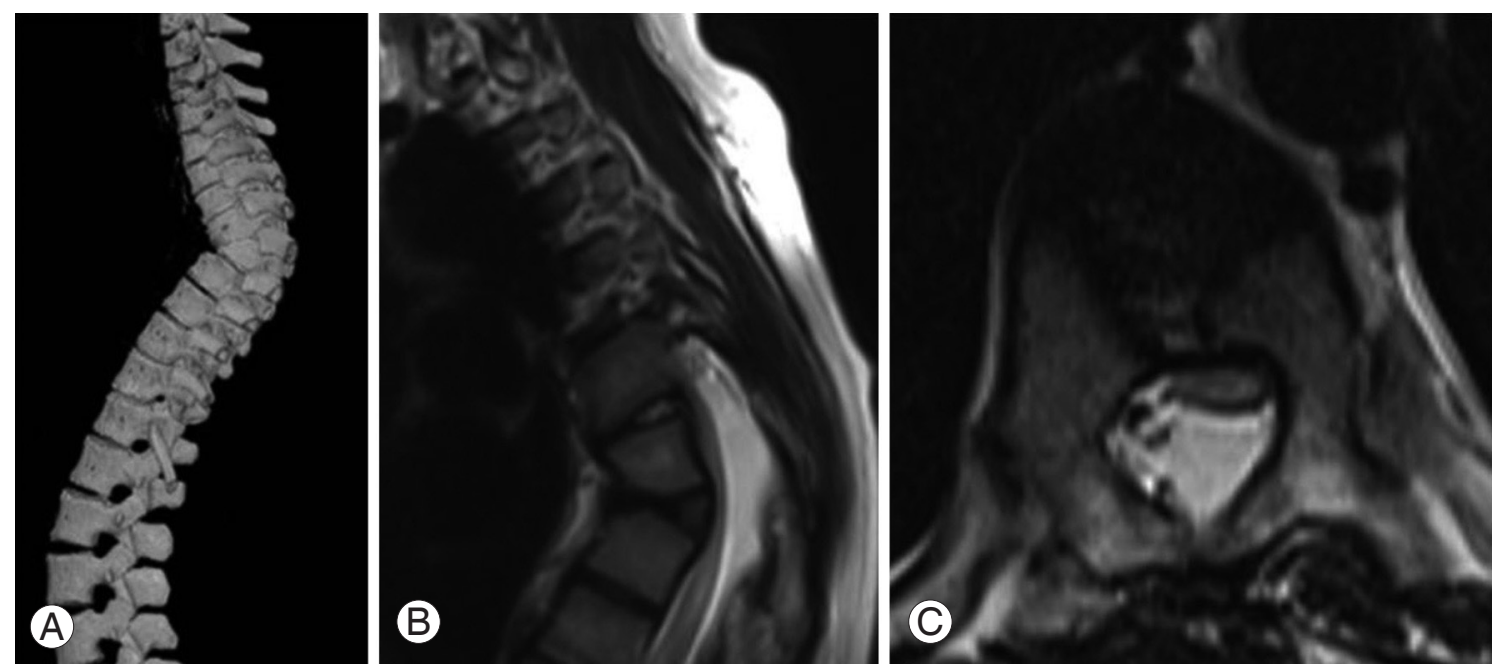

Fig. 1. Preoperaive computed tomography scan (A) showing 3D reconstrution of whole spine wherein kyphotic deformity at $\mathrm{T} 7$ hemivertebra can be visualized. The T2 weighted magnetic resonance imaging (B, C) shows an overstretched flattened cord with signal changes over kyphotic deformity with $\mathrm{T} 7$ hemivertebra in sagittal and axial cut.

$69.5^{\circ}$. The computerised tomography (CT) scan revealed a kyphoscoliotic spine with T7 hemivertebra (Fig. 1A). Her paraparesis had worsened and the neurologic examination revealed some weakness in the ankle dorsilfexors of both the sides (left $>$ right). Knee jerk was exaggerated on both the sides. Sensations were preserved in both the lower limbs. She had a left sided spastic gait and had difficulty in balancing while walking. The MRI revealed a flattened cord with signal changes over the apex of kyphotic deformity with $\mathrm{T} 7$ hemivertebra (Fig. 1B and 1C). In the wake of these developments, she was advised a deformity correction surgery along with anterior decompression to which the parents of the child agreed to.

A surgical protocol in the form of posterior correction of

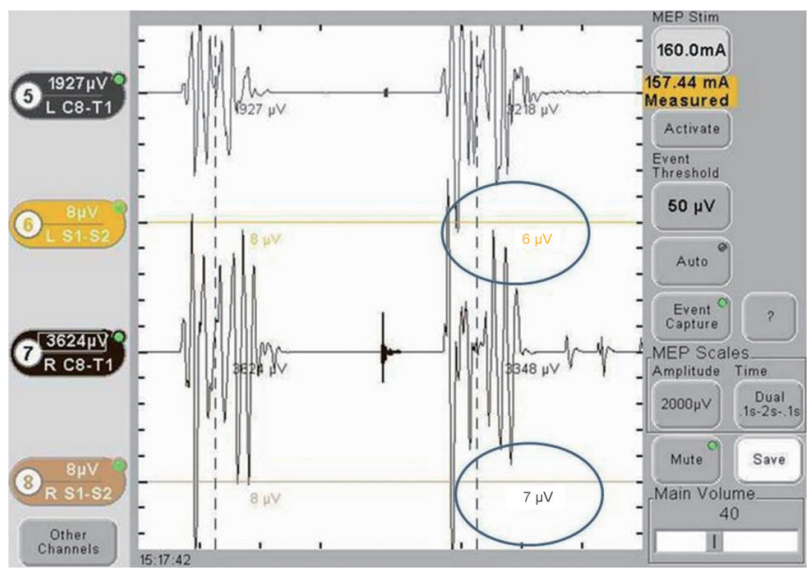

Fig. 2. The trans cranial electrical motor evoke potential recordings while carrying out excision of hemivertebra reveal a significant drop to $6 \mu \mathrm{V}$ on left side and to $7 \mu \mathrm{V}$ on right side. the kyphoscoliotic curve with instrumentation (monoaxial pedicle screw system), posterior decompression, posterior to anterior excision of $\mathrm{T} 7$ hemivertebra, spondylodesis and anterior interbody fusion was planned for her. After facetectomy from $\mathrm{T} 3$ to $\mathrm{T} 10$, posterior instrumentation was done from T3 to T10 sparing T7. The instrumentation was followed by posterior decompression by partial hemilaminectomy T6 and bilateral total laminectomy T7. While carrying out the T7 hemivertebra excision, a trans cranial electrical motor evoke potential evaluation was carried out and it showed a near $100 \%$ drop in both the lower extremities (Fig. 2). Following the condition, the surgery was stopped, and a calculated dose of methyl prednisolone was administered intravenously and a wake up test was called for. Wake up test elicited weak (less than grade 5) movements of both the ankles and great toes. In the wake of a weakly positive wake up test, the surgery was not finished to its conclusion. The hemivertebra excision was discontinued and the deformity was fixed in situ with short rods (T5, T6, and T8) without further correction of the deformity or anterior interbody fusion (Fig. 3A).

Patient was shifted to intensive care unit, where the steroid therapy was continued besides routine supportive medication. On first postoperative day, CT scan revealed an incompletely decompressed $\mathrm{T} 7$ hemivertebra with its posterior cortex intact and kyphotic deformity persisting (Fig. 3B-3D). Thereafter, a gradual decline in the neurologic status was observed. On second postoperative day, power in all the muscle groups of right lower extremity was grade 4 
and in that of left lower extremity was grade 3 . On the third postoperative day, power decreased to grade 2 in right lower extremity and grade 1 in the left lower extremity. On MRI, a flattened spinal cord over the kyphotic deformity along with signal changes and a localized hematoma posterior to the cord were observed (Fig. 3E-3H). The neurologic deterioration continued and neurology became grade 0 in all the groups of muscles (both lower extremities) on fourth postoperative day. An exploratory surgery was performed and the minimal hematoma was drained. Contrary to the MRI findings, the hematoma was not found compressing the cord. Yet in the following period, there was no improvement in the neurologic status. Three weeks later, the short rods were replaced with proper length rods (from T3-T10) and anterior column reconstruction and interbody fusion (T6-T8) with titanium mesh cage and auto lamina bone graft were carried out (Fig. 4). Thereafter, the patient was discharged and she reported to our branch hospital for rehabilitation and physiotherapy.

\section{Discussion}

Sudden onset of paraplegia after kyphoscoliosis correction is a well-known fact, but in the present report, we describe a case wherein unusual gradual deterioration in the neurologic status was observed post deformity correction surgery. We try to analyze the cause(s) that could have led to this gradual neurologic deterioration. Direct mechanical trauma to the cord during the surgery could have triggered off the neuronal apoptotic changes, which we tried to re-
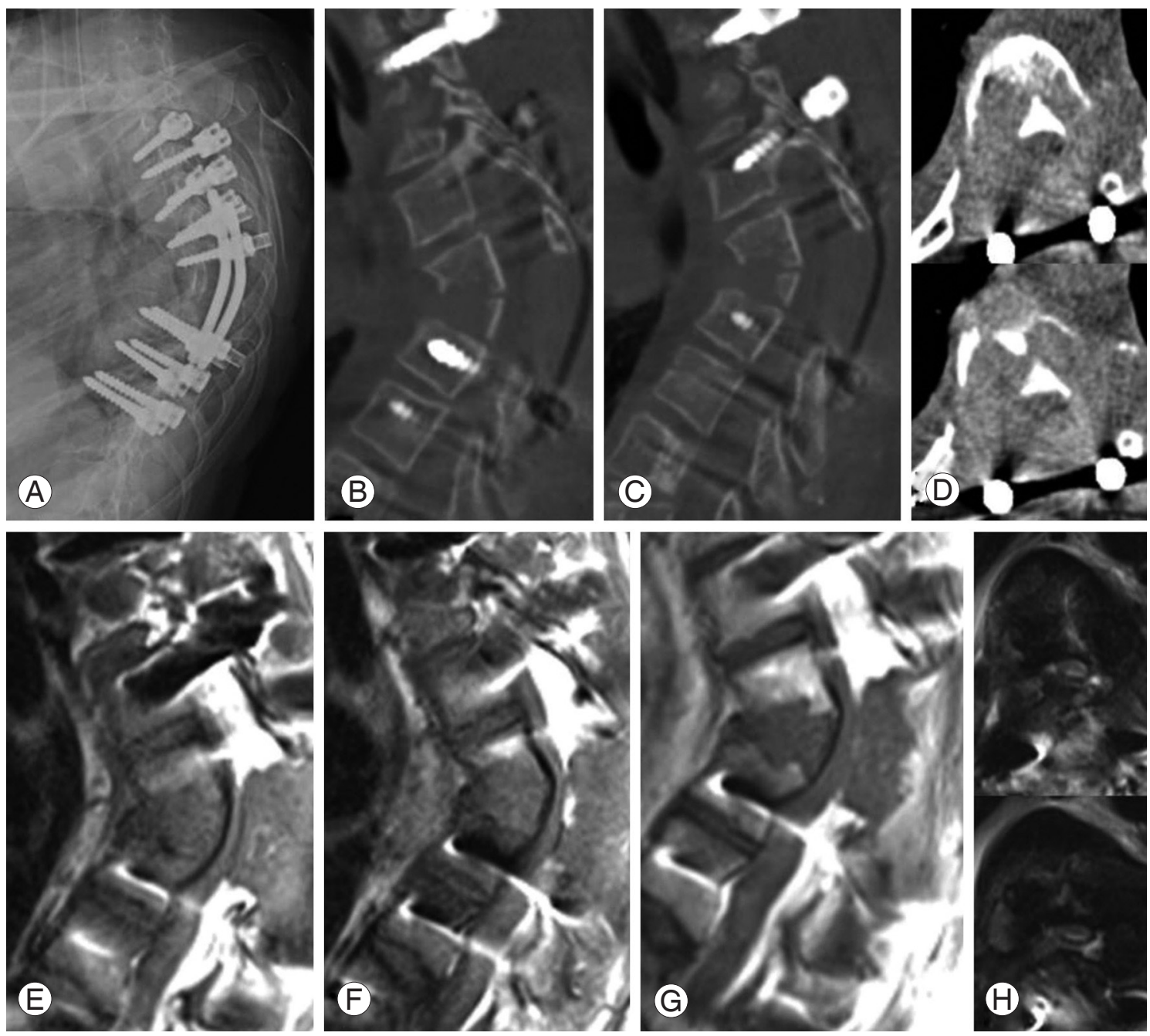

Fig. 3. Immediate postoperative radiograph (A) shows a T3 to T10 instrumented spine with short rods with incomplete excision of T7 hemivertebra. Postoperative computed tomography scan (B-D) on first postoperative day confirms a partially excised T7 hemivertebra with persisting kyphotic deformity. The magnetic resonance imaging $(\mathbf{E}-\mathbf{H})$ done on third postoperative day shows a tense flattened cord with signal changes over intact posterior cortex of T7 and a local hematoma posterior to the cord. 
verse with a mega dose steroid therapy but the phenomenon of progressive damage to the neuronal tissue after the initial injury to the spinal cord could have resulted in the gradual deterioration on neurologic status and finally resulted in the paraplegia [3]. According to a study, it is suggested that severity of anterior angulation of spine is proportional to the pressure within the canal, which in turn may cause vascular insufficiency to the cord as it increases, thereby leading to an altered neurology [4]. Another experimental study suggests that kyphotic angle leads to flattening of the cord over the deformity thereby causing demyelination of anterior funiculus, neuronal loss and atrophy of anterior horn and decreased vascularity at the ventral side of compressed spinal cord [5]. Moreover, while attempting the deformity correction and anterior decompression through single posterior approach, chances of injury to the neural structures increase due to over traction of the dura for better visualization of the structures [6]. In a review study, it was shown that kyphotic deformities compress the cord anteriorly and unless some confirmed posterior pathology exists; posterior canal exposure should be avoided as it renders the spine unstable and increases the kyphotic deformity [7]. A kyphotic spine should be decompressed anteriorly so that the tense flattened cord translates into the vertebral body thereby achieving decompression [8]. Compression to neurovascular structures due to localized hematoma can be a possible reason for neurologic deterioration.

In this particular case, we believe that due to incomplete excision of the hemivertebra, the posterior cortex did not yield when the compression was applied to the screws

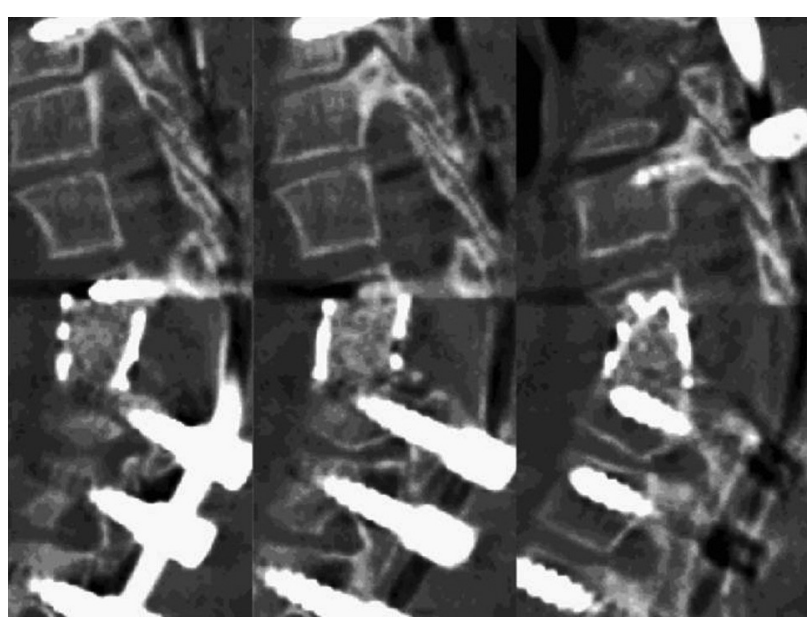

Fig. 4. The final surgery carried out three weeks later, wherein short rods were removed and proper sized rods along with anterior interbody fusion T6-7-8 with titanium mesh cage and auto lamina bone graft were implanted. adjacent to the kyphotic apex. As a result, the correction hinge was shifted to the posterior cortex instead of it lying anteriorly. Moreover, the unyielding posterior cortex offered double hinges, as explained by our schematic representation (Fig. 5) and the compressive force caused the distraction at the adjacent vertebrae rather than collapsing the column. This distraction might have led to the stretching of the spinal cord thereby causing the neuronal damage and hence a deteriorated neurology. The use of monoaxial screws aggravated the distraction, as the tulips were pulled towards the rod and thereby adding to the distraction. The postoperative restriction of patient being in supine position could have added on to the distraction as the fixation was over shorter segment and was not enough to hold the spine in a fixed position. Previously, it has been reported that mechanical stresses due to distraction, compression (more than acceptable limits) or translation etc may result in further neurologic damage [9]. Acute neurologic deterioration after kyphoscoliosis correction surgery is a well-known phenomenon but gradual neurologic deterioration post kyphoscoliosis correction surgery though is a rare phenomenon. In a case report publication, Keyoung et al. [10] tried to evaluate the possible causes of delayed deterioration of neurology after thoracic kyphosis correction surgery and favoured the gradual vascular insult or a local hematoma as the possible causes of such outcome in their case. Our view about depressed neurology in this

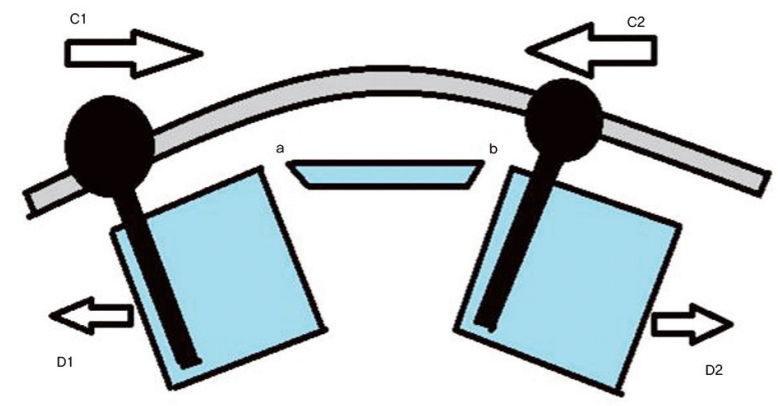

Fig. 5. This drawing illustrates our postulate of cord stretching despite the cantilever beam fixation. In this case, the correction hinge, which ideally would have been at the anterior cortex of excised hemivertebra, was shifted to the posterior cortex. When compression force $(\mathrm{C} 1, \mathrm{C} 2)$ was applied, the partially excised posterior cortex did not yield and offered two hinge points ' $a$ ' and ' $b$ ' and hence a distraction (D1, D2) was observed at the adjacent vertebrae. This caused the stretching of the neural elements. Moreover, the use of monoaxial screws did not allow the translation of the screw tulips over the rod and hence the vertebral movement towards the rod added to the phenomenon of cord stretching. 
particular case is the mechanical forces that caused the post surgery gradual neurologic deterioration. Other remote probabilities for this catastrophe could be a direct trauma to the cord followed by progressive damage to the neural tissue after the initial injury or in the least a vascular insult to the cord.

There is no accurate method for predicting an unfavourable outcome such as postoperative deteriorated neurologic status after deformity correction surgery and despite all the precautions and preventive measures, unexplained deterioration of neurology can occur and the spine surgeons should be aware of this phenomenon.

\section{REFERENCES}

1. Døssing KV, Christensen KS, Thomsen K, Bünger CE. Congenital kyphoscoliosis complicated by paraplegia. Ugeskr Laeger 1995;157:451-3.

2. McMaster MJ, Singh H. The surgical management of congenital kyphosis and kyphoscoliosis. Spine (Phila Pa 1976) 2001;26:2146-54.

3. Bramlett HM, Dietrich WD. Progressive damage after brain and spinal cord injury: pathomechanisms and treatment strategies. Prog Brain Res 2007;161:125-41.

4. Masini M, Maranhao V. Experimental determination of the effect of progressive sharp-angle spinal deformity on the spinal cord. Eur Spine J 1997;6:89-92.

5. Shimizu K, Nakamura M, Nishikawa Y, Hijikata S, Chiba K, Toyama Y. Spinal kyphosis causes demyelination and neuronal loss in the spinal cord: a new model of kyphotic deformity using juvenile Japanese small game fowls. Spine (Phila Pa 1976) 2005;30:2388-92.

6. Zhang J, Shengru W, Qiu G, Yu B, Yipeng W, Luk KD. The efficacy and complications of posterior hemivertebra resection. Eur Spine J 2011;20:1692-702.

7. Lonstein JE, Winter RB, Moe JH, Bradford DS, Chou SN, Pinto WC. Neurologic deficits secondary to spinal deformity. A review of the literature and report of 43 cases. Spine (Phila Pa 1976) 1980;5:331-55.

8. Shenouda EF, Nelson IW, Nelson RJ. Anterior transvertebral transposition of the spinal cord for the relief of paraplegia associated with congenital cervicothoracic kyphoscoliosis. Technical note. J Neurosurg Spine 2006;5:374-9.

9. Chang KW, Cheng CW, Chen HC, Chen TC. Correction hinge in the compromised cord for severe and rigid angular kyphosis with neurologic deficits. Spine (Phila Pa 1976) 2009;34:1040-5.

10. Keyoung HM, Kanter AS, Mummaneni PV. Delayedonset neurological deficit following correction of severe thoracic kyphotic deformity. J Neurosurg Spine 2008;8:74-9. 